\title{
A RECONSIDERATION OF CUP ARTHROPLASTY OF THE HIP
}

\section{With a Precise Method of Concentric Arthroplasty}

\author{
J. CRAWGORI) ADAMS, IONION, EN(iLANI)
}

The contribution made to hip surgery by smith-Petersen's (1939) introduction of cup' arthroplasty is universally acknowledged. A successful arthroplasty, with abolition of pain and preservation of useful movement, is gratifying to the surgeon and a merciful relicf to the patient. Yet the more results one sees, the more does it become clear that in a disconcerting proportion of cases the patient continues to have pain in the hip or thigh after the conventional cup arthroplasty. Because of this and other disadvantages - such as the long period of post-operative rehabilitation that is necessary-many surgeons have abandoned cup arthroplasty in favour of replacement arthroplasty. (Judet and Judet 19:00) which has given promising short-term results and which demands a relatively short period of rehabilitation.
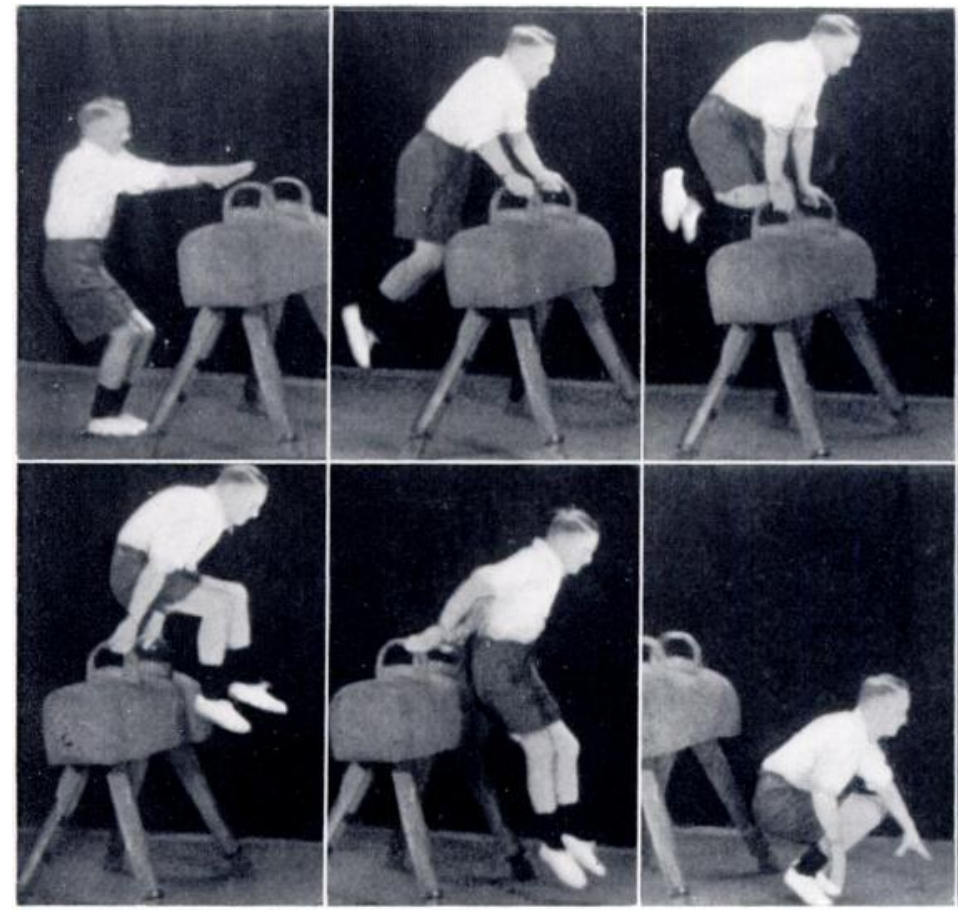

FIG. 1

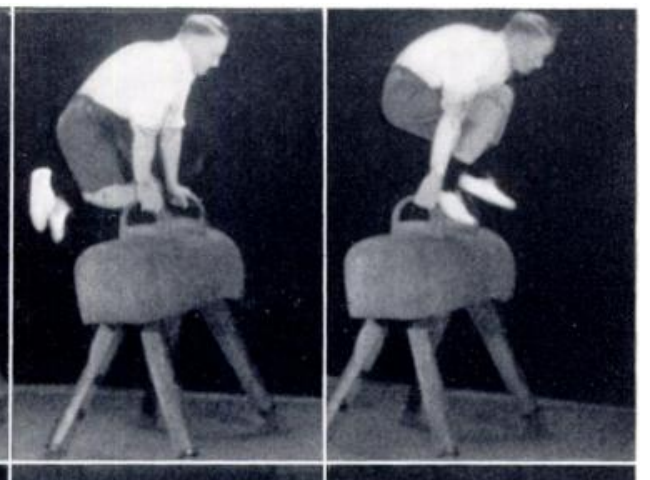

Film cuts to show the excellent function that follows a successful cup arthroplasty. The patient. at fifty-three, has no difficulty in clearing the "horse" with a running jump, bringing his feet forwards either within or outside the hand-grips. His hip does not limit the distance that he can walk or run. He is no longer conscious of any disability. Can a result such as this be achieved and maintained after prosthetic replacement arthroplasty :

Nevertheless most surgeons will agree that a successful cup arthroplasty is as good as, and probably better than, prosthetic replacement of the femoral head; for the function of the hip may be so nearly normal that the patient is no longer conscious of any disability (Fig. 1). Moreover, once a good result has been secured from cup arthroplasty there is every prospect that it will continue to be good for an indefinite period; in fact the function of the 
new joint will usually improve as the years go by. There is movement at both sides of the cup and each movable surface is protected by a smooth lining which ultimately takes on the characteristics of articular cartilage (Smith-Petersen 1948). There is nothing to go wrong; nothing to loosen. On the other hand there is, so far, no indication what will be the long-term results of prosthetic replacement arthroplasty. It may indeed be that the early satisfactory results will be maintained indefinitely. But it may happen that in some cases the prosthesis will loosen on the femur and that further symptoms will eventually arise, whether it be after five, ten or even twenty years. A further disadvantage of replacement arthroplasty is that, if it turns out to be unsuccessful, alternative measures, such as arthrodesis, are rendered difficult by loss of the femoral head.

For these reasons we should not abandon cup arthroplasty. On the contrary, we should consider afresh the causes of persistent pain after unsuccessful cup arthroplasty, and strive to eliminate them. If, as the writer believes, the operation can be made more reliable, it should continue to find a useful place alongside the newer method of prosthetic replacement arthroplasty. In particular, cup arthroplasty would be indicated in relatively young and active patients (provided the local anatomical conditions were favourable) whereas prosthetic replacement would find its main application in the aged or decrepit.

\section{CAUSES OF PAIN AFTER CONVENTIONAL CUP ARTHROPLASTY}

Experience suggests that pain persisting after the conventional operation is not directly correlated with any known variation in the extent of the operation or with the omission of any detail of technique. For instance, the assertion that relief of pain is dependent upon thorough excision of the joint capsule cannot be substantiated: in most of the writer's cases the capsule has been preserved and yet pain has been relieved. It seems more likely that pain after operation results from some unpredictable imperfection in the mechanics of the new joint. That the joint is indeed crude and potentially ill-fitting is clear from the fact that no reliable criteria for selecting the best size of cup have ever been given. The fitting of the cup has always been largely a matter of guesswork and therefore a possible source of error.

Mechanical imperfections of the conventional cup arthroplasty-The usual type of arthroplasty, with cups of the accepted Smith-Petersen pattern, is mechanically inefficient in several respects.

Excessive clearance between the moving parts - When the joint surfaces have been adequately shaped and smoothed the femoral head is usually much smaller than the acetabulum, and the disparity is not fully compensated by interposition of the thin walls of the cup (Fig. 2). Therefore either the head fits loosely in the cup or the cup fits loosely in the acetabulum. A loose fit gives the following disadvantages. 1) There is a tendency to instability with risk of dislocation. 2) The centre of the femoral head may not remain in a constant position within the acetabular cavity in all positions of the joint (Fig. 3). In other words movement may be eccentric, not concentric. 3) The pressure of weight-bearing is not distributed over a wide area of the joint surfaces as in a normal hip, but is concentrated upon the small area of contact (Figs. 2 and 3).

Displacement of the axis of movement-The natural attrition of the articular cartilage and the operative trimming and shaping of the joint surfaces combine to produce a loss of depth, which is restored only partly by the interposition of the thin-walled cup. Consequently the head of the femur is displaced upwards and the abductor muscles are shortened (Fig. 2).

The shape of the cup-The straight sides and everted rim of the cup hinder its free concentric movement in the acetabulum; rotation about its central axis is the only movement that can occur concentrically.

Observations at re-exploration for pain after cup arthroplasty-Three unfavourable features have been observed. The commonest is eccentric movement: when the joint is moved through its full range the axis of movement of the femoral head does not remain 
constant in relation to the acetabulum. Sometimes, at a certain point in the morement the femoral head will suddenly slip into another part of the cup. This observation bears out what was suspected from mechanical considerations. The second unfavourable feature that has been found is local exposure of bone, usually on the femoral head at the point of weight bearing. The exposed bone, hard and vellow, contrasts strikingly with the smooth lining membrane that covers the rest of the articular surface and which, in cases of painless arthroplasty, has been observed to cover the whole articular surface (cibson and llilliams

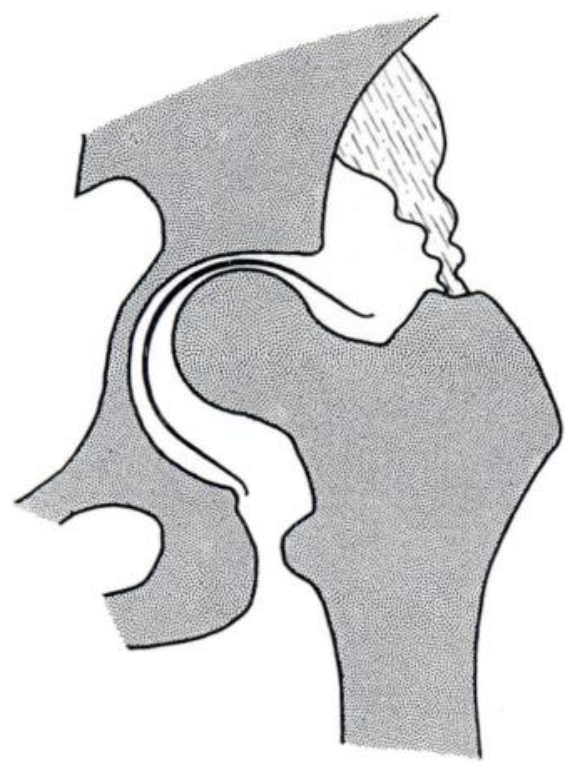

lig. 2

Diagram showing the mechanical deficiencies of the conventional cup arthroplasty-- namely. excessively loose fit, upward displacement of the femur, and incongruous shape of the cup. Note that the pressure of weight-bearing is concentrated on a small area of the femoral head (see te.st).

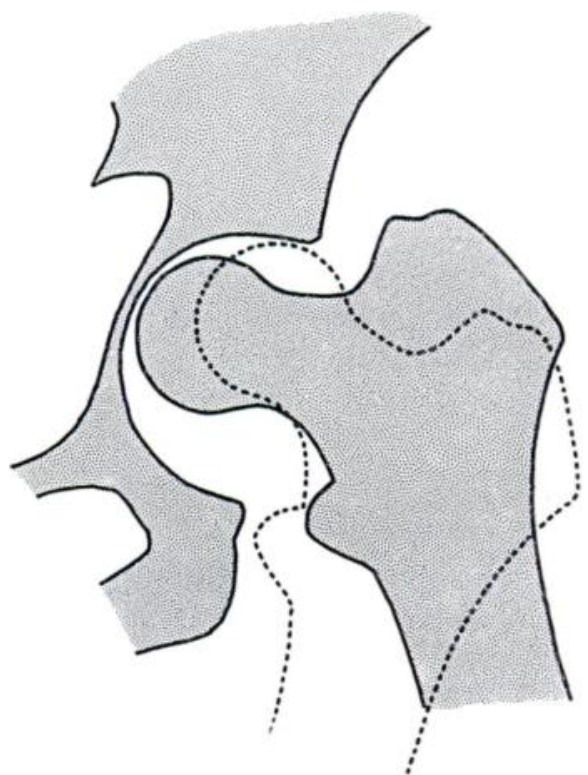

Fili: 3

To show how a loose fit favours eccentrie movement. There is nothing to retain the axis of mosement of the femoral hearl in a constant relationship to the acetabulum in all positions of the joint. Here for example, the axis has moved outwards between abduction fentinuous line) and adduction (broken line). ("up) omitted for clarits.

19.5l). The third feature, seen especially when the head and neck of the femur are short, is abutment of the rim of the cup against the soft-tissue coverings of the bone in the trochanteric region.

Interpretation of the findings - Eccentric movement due to ill-fitting joint surfaces must predispose to recurrent strains of the capsular ligaments (whether natural or regenerated). The action of such a joint may be likened in principle to that of a cam, a device used widely in engineering when powerful tension is repuired. Though eccentric movement is probably the predominant cause of strain, inefficiency of the abductors, from upward displacement of the femur, may be a contributory factor because it throws the stress of checking adduction upon the capsular ligaments. Recurrent strains of the capsule cause sharp local pain on movement. In addition, the mechanical irritation sets up an inflammatory reaction with local congestion of the tissues and conseguent aching which may persist even at rest.

The cause and the significance of local areas of exposed bone at the articular surfaces are debatable. When there is marked inequality between the moving parts (femur, cup), acetabulum) the pressure of weight-bearing is concentrated in a very small area (Fig. - 2) and it is reasonable to suppose that excessive local pressure may prevent the development of a stable protective membrane upon the bone. When a deficiency in the protective membrane is associated with pain the strong possibility that the two are correlated must be accepted.

VOL. $35 \mathrm{~B}$, NO. 2, MAY 1953 
The significance of impingement of the rim of the cup at the base of the femoral neck is also uncertain. It is probably less important as a cause of pain than the other factors mentioned.

Conclusions - It is believed that pain after the standard cup arthroplasty arises from three cause's, in the following order of importance. 1) Recurrent strains of the capsular ligaments. permitted by eccentric movement of the femoral head in the acetabulum and aggravated by inefficiency of the alductor muscles. 2) Local exposure of bone in the weight-bearing area of the joint, faroured by disparity in size between the moving parts and consequent concentration of pressure in a small area. 3) Impingement of the rim of the cup at the baste of the femoral neck, when the head and neck are short.

\section{A PRECISE METHOD OF CONCENTRIC ARThroplasty}

Believing that pain after cup arthroplasty often arises from the mechanical imperfections described, the writer has developed during the last three years a modified technicue which aims 1) to ensure concentric movement of the femoral head within the acetabular cavity; 2) to allow only a small but a uniform clearance between the moving parts; and 3) to retain the axial point of the femoral head as near to its normal position as possible. The principal modifications are in the design of the cup and in the careful shaping of the joint surfaces to exact dimensions (Fig. 4). The cup forms approximately two-thirds of a sphere (Fig. 5). Its walls are a quarter of an inch thick - sufficient to compensate for loss of bone and cartilage and thereby to minimise upward displacement of the femoral head. The inside of the cup is machined to a spherical contour. When the femoral head is within the cup there is a clearance between their surfaces of one-sixteenth of an inch all round; this is sufficient to allow the formation of a smooth surface membrane, but not enough to impair stability. A similar clearance is allowed between the cup and the acetabulum. Only one size of cup has been used, but a second size is available for very small hips. Since the new

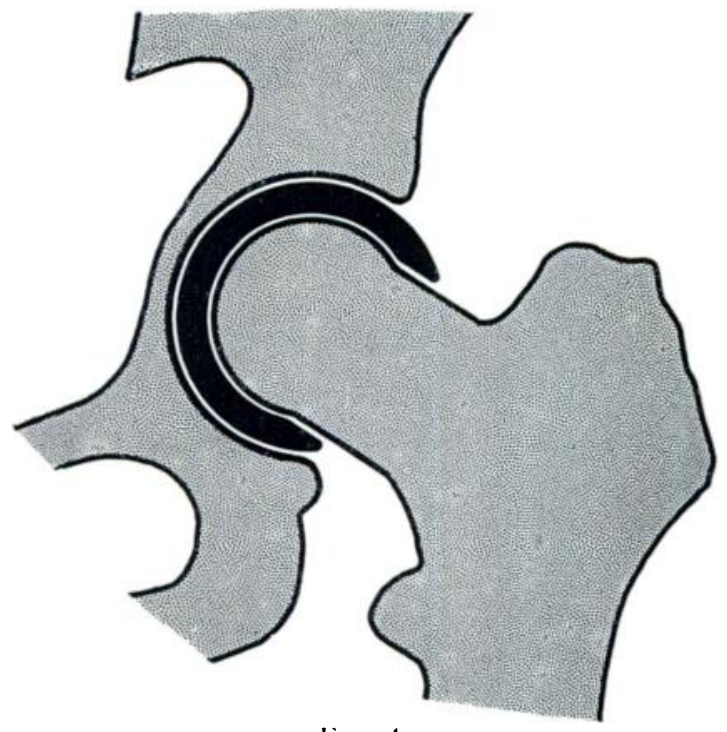

Fici. 4

Sicale drawing of concentric arthroplasts. Note the snum tit of the moving parts, which ensures concentric movement and widely distributed weight-bearing contact. The clearance is sufficient to allow the formation of lining membranes upon the acetabular and femoral surfaces. The thickness of the cup compensates for the loss of bone and cartilage and thus minimises upward displacement of the femur. joint is made to exact measurements, difficulty in selecting the correct size of cup is eliminated. The cups used so far have been of acrvlic resin, hut cups of inert metal have recently been introduced.

Anatomical requirements - The head and neck of the femur should be of good length. The bony structure of the femoral head and of the acetabular region of the ilium should be sound, and the joint must be free from active infection. Inless these conditions are satisfied some other procedure would usually be preferred to cup arthroplasty.

Technique of operation The operation follows the general lines of the smithPetersen procedure. The incision will depend upon individual preference: the writer prefers a postero-lateral approach, which gives more direct access to the acetabulum than do the anterior incisions. After dislocation of the hip the diameter of the femoral head is measured with a caliper: if it is greater 


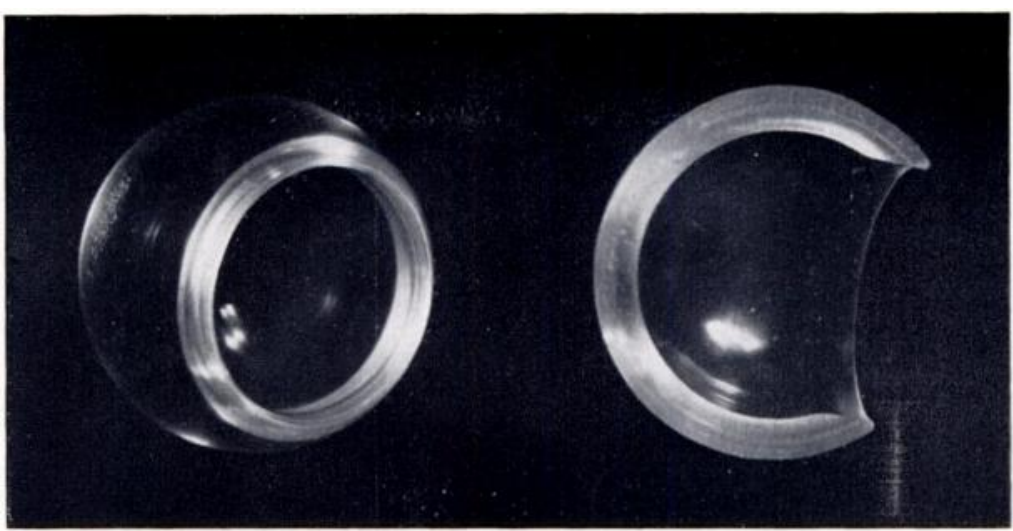

FIG. 5

The modified cup shown entire and sectioned.

than 1 inches the larger size of cup is the one to use. This will almost always be the case, except in diminutive women (arthroplasty has not been undertaken in children). The acetabulum is enlarged concentrically by suitable gouges, all diseased and sclerotic bone being removed. It is important that in removing bone the carity be kept spherical. Enlargement proceeds until the brace-operated ball reamer $(\geq 36$ inches in diameter at efuator, or $1 ;$ inches if the smaller cup is to be used) will just fit into the carity. The reamer is used only for the final smoothing, not to form the carity. This point should be emphasised. The reamer removes very little bone, and if it is used before the cavity has been made large enough it will not cut its way to the full depth of the acetabulum, even though considerable time and effort be expended. In that event the carity will not be spherical but will be shaped as shown in section in Figure 6 , and in conseguence the cup will not fit deeply enough. When the acetabulum has been fashioned the femoral head is shaped with a chisel and finally smoothed with a reamer to a spherical contour, the diameter being 19 inches ( $1 \frac{1}{4}$ inches if the smaller cup is used). The neck is shaped to a diameter a little smaller than that of the head (Fig. 4). (Care must be taken to ensure that the head is not cut too small: the narrowed mouth of the cup should push over the head only with considerable force (once in position, however, the cup will rotate freely on the head). When the dislocation has been reduced the joint is remarkably stable and movements are concentric. When this has been confirmed the capsule is repaired and the wound closed. The origins of the adductor muscles are divided subcutaneously as a routine, either before or at the end of the operation. Post-operative treatment - The joint is so stable that dislocation is not to be feared and the details of the early post-operative treatment are relatively unimportant. The writer prefers to rest the joint in a comfortable position such as that afforded by Russell traction, encouraging static muscle contractions but deferring joint movements until two or three weeks after the operation, when the soft tissues may be presumed to be healed. Thereafter, active and assisted hip movements are encouraged, with particular attention to the redevelopment of the extensor and abductor muscles. Walking is begun after six or seven weeks, according to progress.

Comment-It must be emphasised that meticulous care is necessary in shaping the acetabulum and the femoral head to the

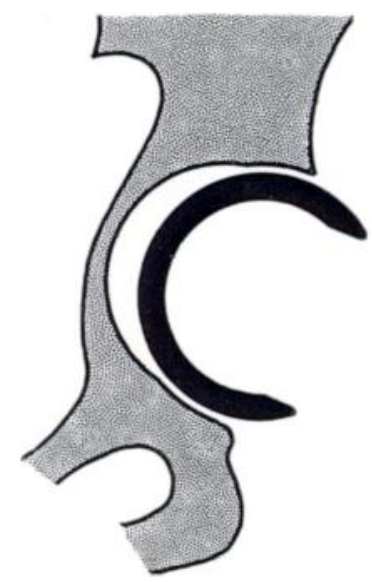

Fici. 6

l)iagram showing the result of attempting tu ream the acetabular catsits before it has been enlarged sufficiently by gouges. The smoothing reamer cuts away very little bone, and if used prematurely it will not cut its way to the full depth of the socket.

VOI. 35 B, No. 2, MAY 1953 


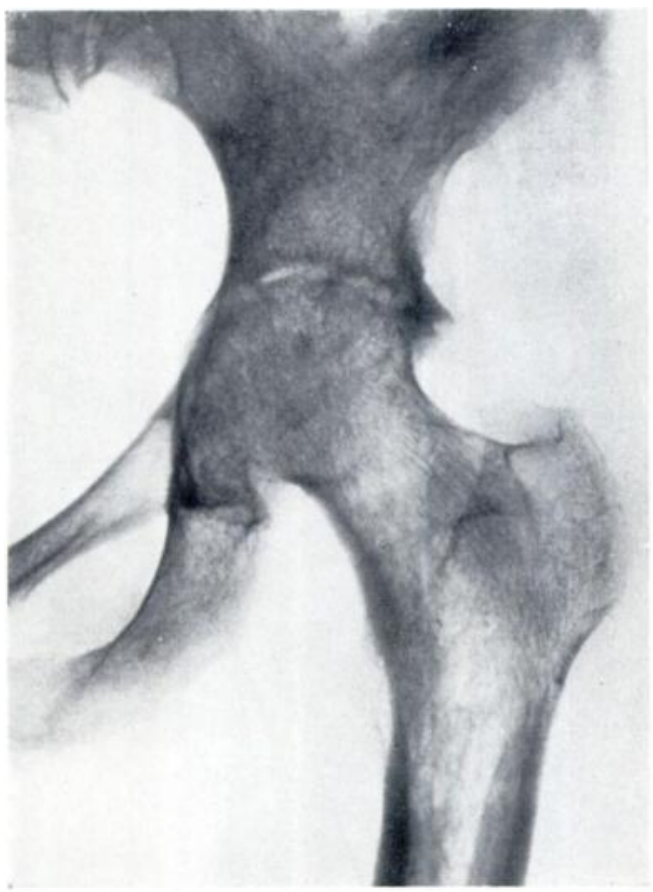

Fili. 7

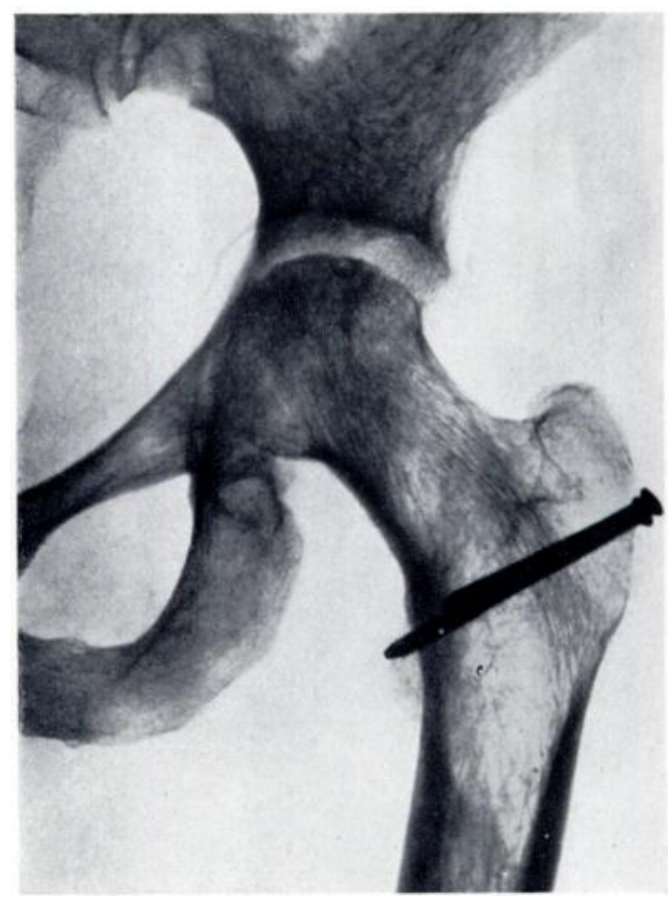

Fili. 8

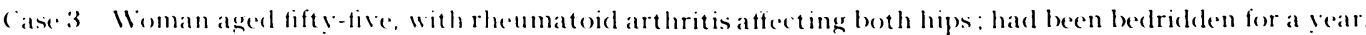
ligure 7 left hip, before operation. Figure 8 (nne vear after concentric arthroplasty. The right hip wits similar. Note that shenton's line is almost unbroken, which suggests that the axis of moxement is approximately in the normal position.

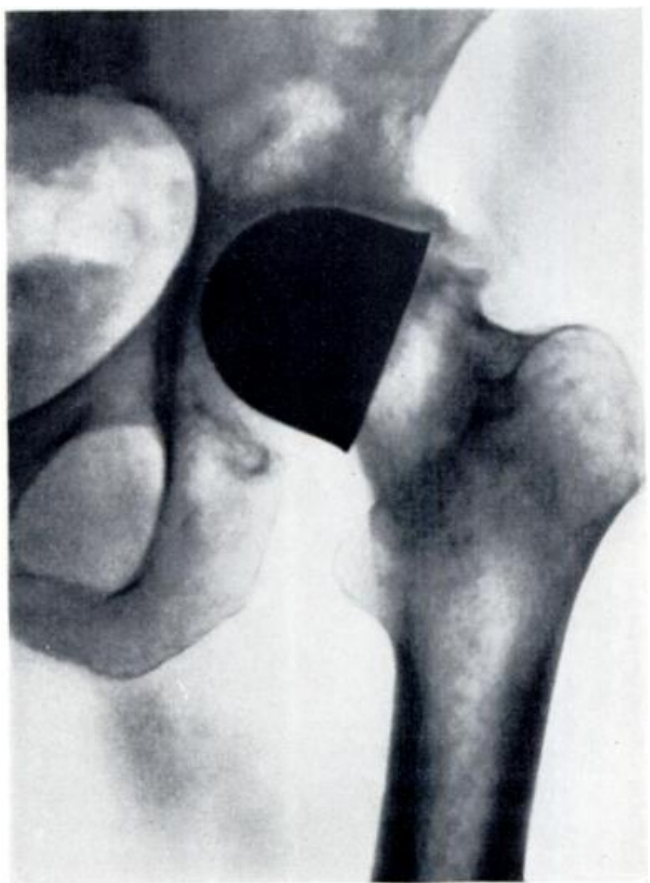

Fis. 9

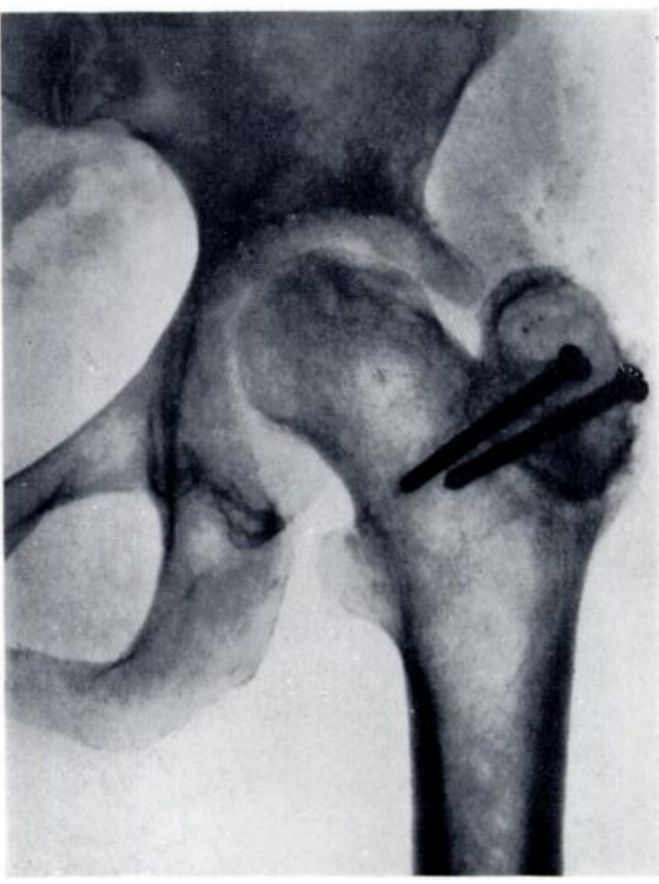

FIG. 10

(ase 5- Woman aged sixty-seven. Previous cup arthroplasty had been done unsuccessfully for painful osteoarthritis. Figure 9 Before re-operation. Figure 10 Six months after concentric cup arthroplasty. 
correct size and shape. The whole object of the method is defeated if the operation is performed hastily without regard to the details of technipue.

\section{CLINICAL ASSESSMENT}

So far this modified technicfue of arthroplasty has been carried out in only twenty-seren cases, and it is therefore too early to assess the results accurately. It can he said

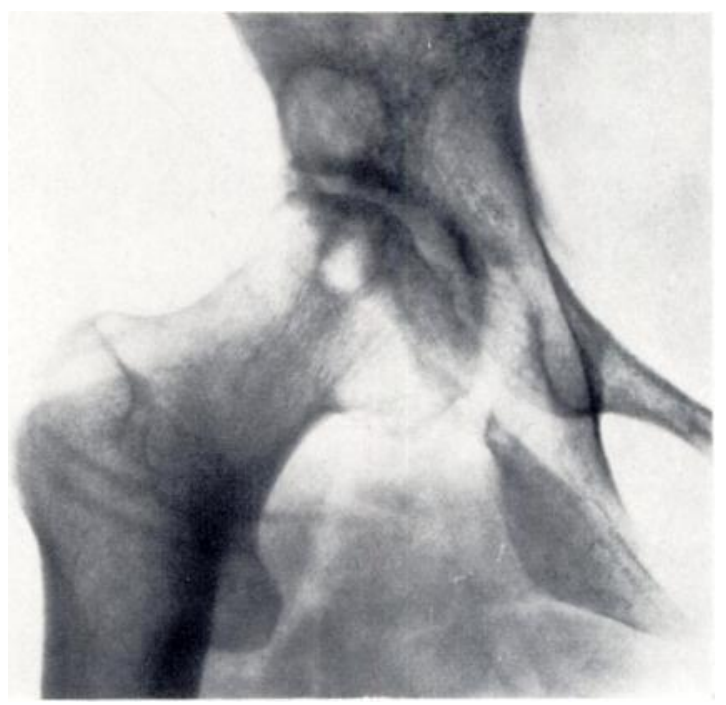

I:11:. 11

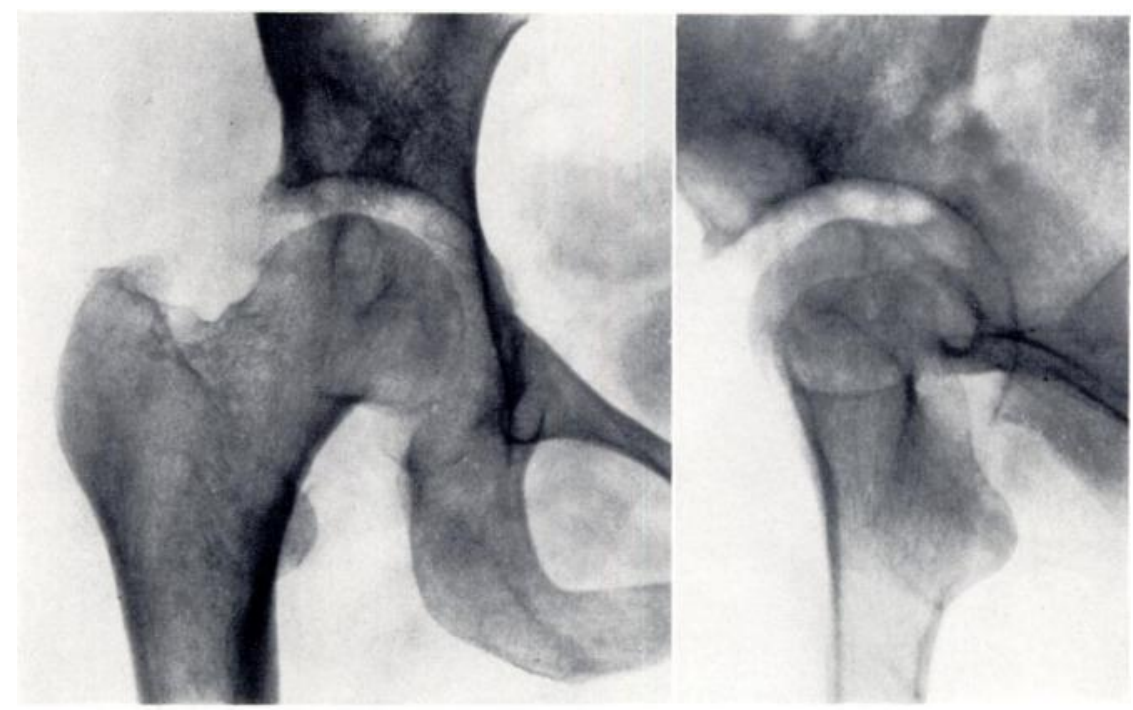

Fic;. 12

Case 18-Woman aged thirty-nine. ()d congenital dislocation of hip treated in early childhood. Lain had become disabling. Figure 11- Before arthroplasty. Figure 12 Nine monthsafterconcentric cuparthroplasty. Fote the concentricoutlines of the joint surfaces.

with confidence, however, that patients have done better after this operation than did the writer's patients after the conventional arthroplasty. Rehabilitation after operation proceeds more rapidly than after the conventional cup arthroplasty, probably because of the greater stability of the new joint, by virtue of which the patient may often regain the power of straight leg raising within three weeks of operation and may walk with fair confidence 
J. C. ADAMS

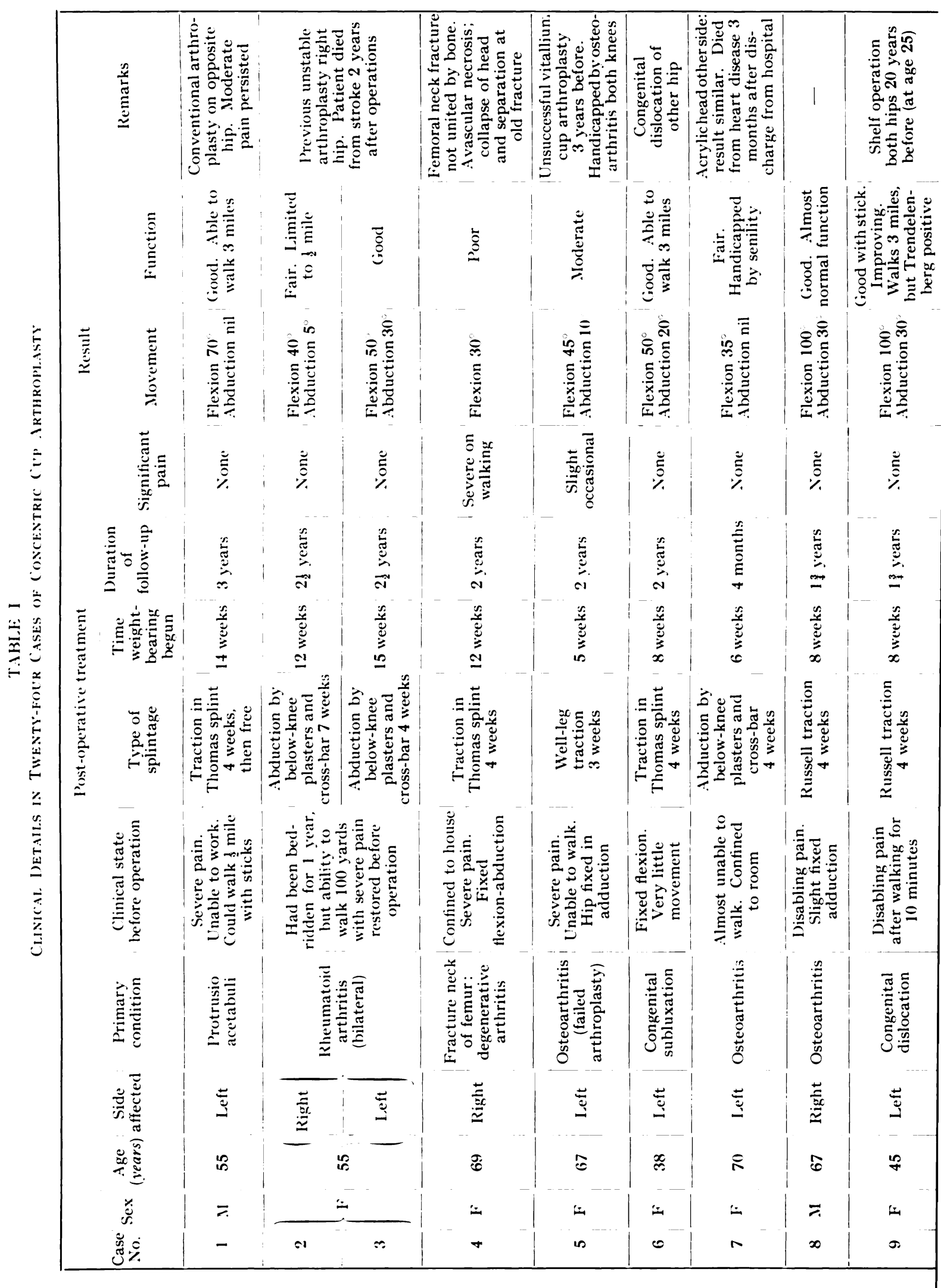

THE JOURNAL OF BONE AND JOINT SURGERY 


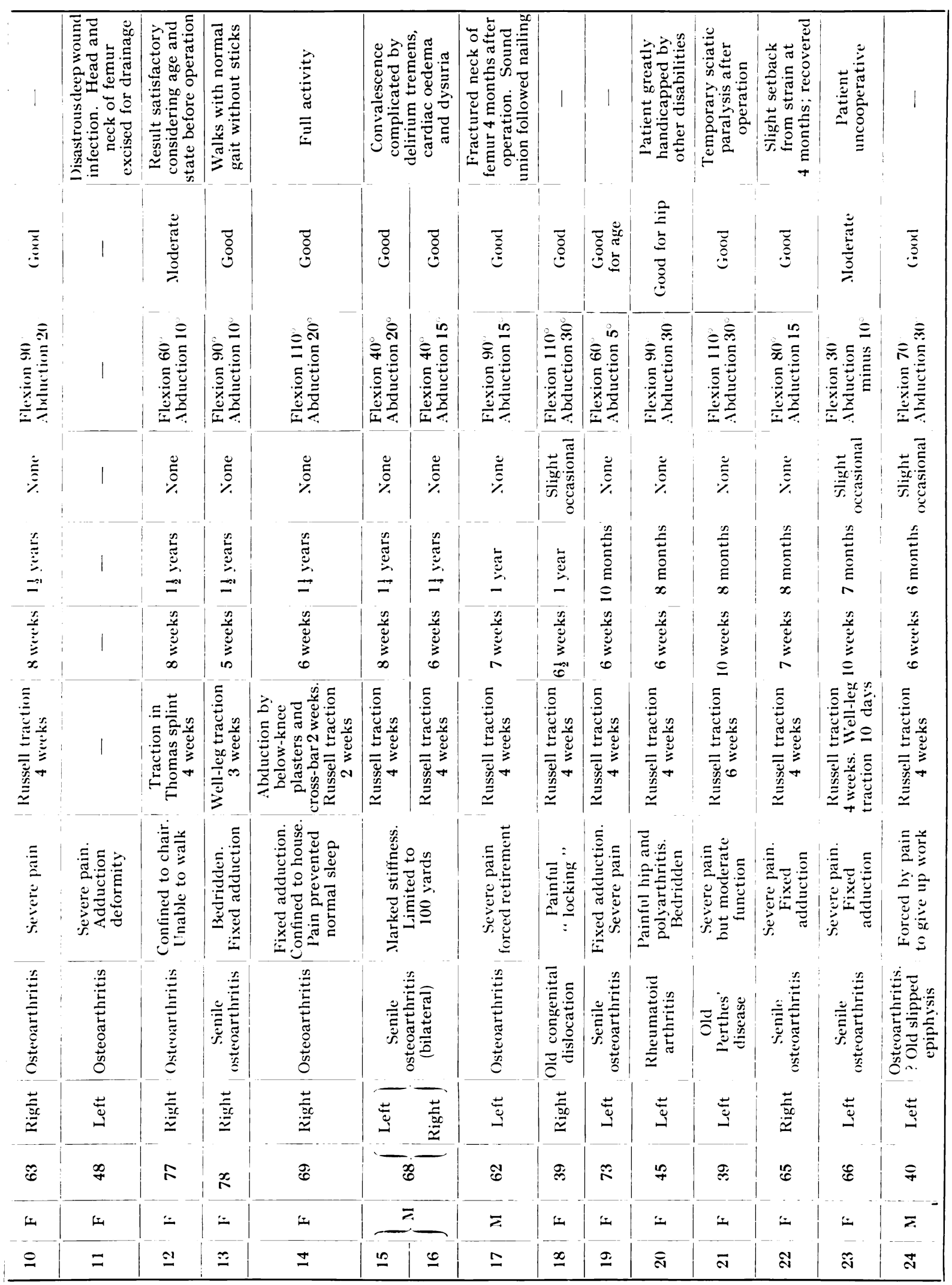

Vol. $35 \mathrm{~B}$, xo. 2, MAY 1953 
after six or seven weeks. In no case has dislocation or subluxation occurred. Clinical details of the twenty-four cases in which the reconstruction was completed more than six months ago are shown in Table I, and illustrative radiographs are reproduced in Figures 7 to $1 \cdot 2$. In one case the reconstructed joint was ruined by a deep wound infection which occurred despite a rigid aseptic technique; excision of the head and neck of the femur was necessary as a life-saving measure. With one exception the remaining patients are free from pain or virtually so. The unsuccessful case was in a woman with old avascular necrosis after fracture of the neck of the femur, which was erroneously thought to be united by bone. The head collapsed within the cup, and displacement occurred at the site of the original fracture. such a case would not now be considered suitable for cup arthroplasty.

\section{DISCUSSION}

It seems reasonable to suggest that a precisely constructed ball-and-socket joint acting concentrically about the normal axis of hip movement is more likely to give consistently good results than a loosely fitted joint that may move eccentrically and about a displaced axis. This supposition is borne out by the writer's present experience of the concentric cup arthroplasty described here. Reconstructive operations upon the hip are in a phase of rapid evolution, and it is not suggested that the method described will represent more than an intermediate stage in the evolution of cup arthroplasty. It seems that it may be an improvement on the older method, but no doubt there will be room for future development, perhaps from further modification in the design of the cup, or from other refinements of technique. The main purpose in presenting this paper has been to stimulate further thought and research towards the perfection of the technique of cup arthroplasty, the fundamental principle of which appears to be sounder than that of prosthetic replacement arthroplasty when prolonged service from the reconstructed joint is demanded.

\section{SUMMARY}

1. The unreliable results of the conventional cup arthroplasty are attributed to mechanical imperfections in the reconstructed joint.

D. If its reliability can be improved, there will remain a place for cup arthroplasty, which, in relatively young and active patients, offers advantages over prosthetic replacement arthroplasty:

3. A technique of concentric cup arthroplasty is described. The new joint is shaped with precision to exact dimensions and lined with a cup designed to ensure stability, concentric movement and a uniform clearance between the moving parts.

4. The results so far are encouraging and justify continued clinical trial.

The cups are made by Messrs Willen Bros. Ltd., 44 New Cavendish Street, London, W. 1, whose courteous cooperation I acknowledge gratefully. I am indebted to Dr Cardew, of the Photographic I)epartment of St Mary's Hospital Medical School, for the photographs.

\section{REFERENCES}

(iibsox. A., and Willians, T. H. (1951): Changes in the Femoral Head underlying a Vitallium Cup. Journal of Bone and Joint Surgery, 33-B, 119.

Judet, J., and Judet, R. (1950): The L'se of an Artificial Femoral Head in Arthroplasty of the Hip Joint. Journal of Bone and Joint Surgery, 32-B, 166.

Simith-Petersex, M. X. (1939) : Arthroplasty of the Hip: a New Method. Journal of Bone and Joint Surgery, $21,169$.

Smith-Peterses, M. N. (1948): Fvolution of Mould Arthroplasty of the Hip Joint. Journal of Bone and Joint Surgery, 30-B, 59. 